INPLASY

PROTOCOL

To cite: $\mathrm{Ba}$ et al. The Predictive Value of blood-based tumor Mutation Burden on Efficacy of Immune Checkpoint Inhibitors in Solid Cancers: A Systematic Review and Meta-Analysis. Inplasy protocol 202110115. doi:

10.37766/inplasy2021.1.0115

Received: 28 January 2021

Published: 29 January 2021

Corresponding author:

$\mathrm{He} \mathrm{Ba}$

bahe2474364@163.com

Author Affiliation:

The First Affiliated Hospital of Anhui Medical University, Hefei 230022

Support: NSFC (81693439).

Review Stage at time of this submission: Preliminary searches.

Conflicts of interest:

None.

\section{The Predictive Value of blood-based tumor Mutation Burden on Efficacy of Immune Checkpoint Inhibitors in Solid Cancers: A Systematic Review and Meta-Analysis}

Ba, $\mathrm{H}^{1}$; Chen, J2; Zhu, Y3.

Review question / Objective: In this systematic review and meta-analysis, the comparison on efficiency of immune checkpoint inhibitors (ICIs) between advanced cancer patients in blood-based tumor mutation burden (bTMB) high and bTMB low group will be conducted to assess the value of bTMB in appropriate groups identifying and prognosis predicting for cancer immunotherapy, and overall subgroup analyses will be carried out to identify potential source of heterogeneity.

INPLASY registration number: This protocol was registered with the International Platform of Registered Systematic Review and Meta-Analysis Protocols (INPLASY) on 29 January 2021 and was last updated on 29 January 2021 (registration number INPLASY202110115).

\section{INTRODUCTION}

Review question / Objective: In this systematic review and meta-analysis, the comparison on efficiency of immune checkpoint inhibitors (ICIs) between advanced cancer patients in blood-based tumor mutation burden (bTMB) high and bTMB low group will be conducted to assess the value of bTMB in appropriate groups identifying and prognosis predicting for cancer immunotherapy, and overall subgroup analyses will be carried out to identify potential source of heterogeneity.

Condition being studied: Immune checkpoint inhibitors (ICIs) have been identified to improve response and survival 
in multiple tumors. However, the efficacy seems unsatisfactory in unselected patients indicating eligible biomarkers are required to identify subgroups appropriate for cancer immunotherapy. At present, increasing researches had demonstrated the predictive role of tumor mutation burden (TMB) in cancer immunotherapy, but tissue samples often provide inadequate DNA for sequencing and may underrepresent tumor molecular heterogeneity. Circulating tumor DNA (ctDNA), shed into blood by tumor cells, is increasingly utilized to identify actionable mutations and predict response to therapy. Herein, this up-to-date systematic review and meta-analysis will be conducted to explore the predictive value of blood-based tumor mutation burden (bTMB), determined by ctDNA-based next-generation sequencing (NGS), on cancer patients treated with immune checkpoint inhibitors.

\section{METHODS}

Search strategy: PubMed, Embase, Cochrane, Web of Science and ClinicalTrials.gov will be searched from inception to 02 February, 2021 for studies published in English by two investigators independently. The search terms are as follows: (PD-1 OR PD-L1 OR CTLA4 OR Ipilimumab OR Nivolumab OR Tremelimumab OR Pembrolizumab OR Lambrolizumab OR Avelumab OR Atezolizumab OR Durvalumab OR "immune checkpoint inhibitor" OR "immune checkpoint inhibitors" OR "ICI" OR "ICls" OR "immune checkpoint blocker" OR "immune checkpoint blockers" OR "ICB" OR "ICBs") AND (mutation burden OR mutational burden OR mutation load OR mutational load OR TMB OR TML) AND (cell free OR circulating OR blood OR plasma OR extracellular OR serum).

Participant or population: Advanced cancer patients (stage IIIB-IV) treated with inhibitors of PD-1/PD-L1, CTLA-4, or their combination and the efficiency of therapy was evaluated by blood-based tumor mutation burden (bTMB) which has cut-off value.
Intervention: ICls treatment (PD-1/PD-L1 inhibitor, CTLA-4 inhibitor or their combination).

Comparator: According to the cut-off value, patients were divided into bTMB-high and bTMb-low groups. The relative efficacy of ICls between two groups measured in terms of objective response rate (ORR), overall survival (OS) and progression free survival (PFS) will be compared.

Study designs to be included: Clinical trials or cohort studies.

Eligibility criteria: (1) Clinical trials or cohort studies assessed inhibitors of PD-1/PD-L1, CTLA-4, or their combination, in patients with cancer, and the efficiency of therapy was evaluated by bTMB which has cut-off value. (2) Odds ratio (OR) of objective response rate (ORR), or hazard ratio (HR) of progression-free survival (PFS) or overall survival (OS), and their $95 \%$ confidence intervals $(95 \% \mathrm{Cl})$ were given in the article (3) The number of patients accessible for evaluation was no less than 20.

Information sources: PubMed, Embase, Cochrane, Web of Science and ClinicalTrials.gov.

Main outcome(s): Overall survival (OS), progression free survival (PFS) and objective response rate (ORR) of patients.

Quality assessment / Risk of bias analysis: The Newcastle-Ottawa Scale (NOS) will be adopted to assess the quality of studies included. The total score ranged from 0 to 9, as 8-9 points indicates high quality of a study, five to seven points indicates medium quality, and points lower than five indicates poor quality.

Strategy of data synthesis: Heterogeneity among individual studies will be evaluated by the $Q$ test; $12>50 \%$ or $P<0.10$ indicates significant heterogeneity. Pooled OR or HR with $\mathrm{Z}$ test will be calculated by randomeffects model. Funnel plots will be constructed, and Begg's test and Egger's test will be performed to evaluate publication bias $(P<0.10$ will be 
considered as visible publication bias). Sensitivity analysis will be used to test the stability of the results in the meta-analysis

Subgroup analysis: Subgroup analyses stratified by cancer type, area of patients, class of immune checkpoint inhibitors, TMB sequencing method, and line of therapy will be conducted.

Sensitivity analysis: (1) Observe changes in the statistical result after studies with the largest and smallest weight are excluded. (2) Synthesis the data with random-effects model and fixed-effects model successively (3) Overall subgroup analyses will also be carried out to identify potential source of heterogeneity.

Country(ies) involved: China.

Keywords: tumor mutation burden, tumor mutational burden, immune checkpoint inhibitor.

Contributions of each author:

Author 1 - He $\mathrm{Ba}$ - literature searching and manuscript writing.

Email: bahe2474364@163.com

Author 2 - Jie Chen - literature searching and data extracting.

Author 3 - Yaodong Zhu - literature

searching and data extracting.

Email: zhuyaodong2013@163.com 